\title{
Insular epilepsy surgery under neuronavigation guidance using depth electrode
}

\author{
Young Seok Park • Yun Ho Lee • Kyu Won Shim • \\ Yoon Jin Lee • Heung Dong Kim • Joon-Soo Lee • \\ Dong-Seok Kim
}

Received: 22 October 2008 / Published online: 13 January 2009

(C) The Author(s) 2008. This article is published with open access at Springerlink.com

\begin{abstract}
Objectives The unfavorable surgical results that have been reported with insular epilepsy surgery may be related to inadequate monitoring. Insular epilepsy surgery requires the precise monitoring and tailored removal of the epileptic zone. The purposes of this study were to precisely monitor the hidden cortex and determine the effectiveness of using different monitoring methods, including subpial depth electrodes.

Materials and methods This is a study of a single center series of six patients (three men, three women) who underwent insular surgery between May 2006 and December 2007. All patients had experienced medically intractable seizures without any evidence of a tumorous condition based on previous examination. We used strip electrode implantation in one patient and stereotactic depth electrode insertion in two patients, which resulted in partial removal of the insular. The other three patients underwent open direct subpial depth electrode insertion, which resulted in total removal of the insular cortex.

Results There were no surgery-related complications with the insulectomy after insertion of the subpial depth electrodes, regardless of the monitoring method or extent of removal. All patients except for one showed considerable
\end{abstract}

Y. S. Park · Y. H. Lee • K. W. Shim • D.-S. Kim $(\bowtie)$

Pediatric Neurosurgery, Pediatric Epilepsy Clinic,

Severance Children's Hospital, Department of Neurosurgery,

Brain Research Institute, Yonsei University College of Medicine,

134, Shinchon-dong, Seodaemoon-gu,

Seoul 120-752, Republic of Korea

e-mail: dskim33@yuhs.ac

Y. J. Lee $\cdot$ H. D. Kim $\cdot$ J.-S. Lee

Department of Pediatrics, Severance Children's Hospital,

Pediatric Epilepsy Clinic, Yonsei University College of Medicine,

Seoul, Republic of Korea improvement in epilepsy (83.3\% Engle class I, 16.5\% Engle class II). In the three patients who had open direct subpial depth electrodes inserted, we achieved total insulectomy without increasing the surgical morbidity, while in the other three patients, the insular removal was only partial.

Conclusion We demonstrate that image-guided insular surgery with safe and accurate depth electrode guidance results in an optimal outcome and complete resection of the insular cortex.

Keywords Depth electrode - Epilepsy · Insular · Intractable epilepsy. Surgery

\section{Introduction}

The insular cortex hidden by the frontal or temporal operculum can be an epileptic zone [3, 14, 15]. Seizures originating in the insular cortex used to be confused with frontal, temporal, or frontotemporal lobe seizures. Unacceptable morbidity and mortality occur with insular cortex removal because of neurovital structures and the associated vasculature [11, 18, 21, 22]. Because of its location deep within the sylvian fissure, scalp electroencephalography is relatively insensitive $[3,24]$. The implantation of subdural electrodes, such as transsylvian or stereotactic depth electrodes, perpendicular to the midsagittal plane by the transopercular route can be used for limited monitoring of the wide insular cortex $[3,14]$.

This confusion related with poor monitoring suggests that proper insular monitoring may be the key to solve insular epilepsy. We therefore hypothesize that precise insular monitoring methods result in good surgical outcome. To determine which monitoring methods in insular 
epilepsy are most effective to diagnose and operate, we used various insular monitoring devices including open direct subpial depth electrodes.

\section{Materials and methods}

\section{Patient selection}

In a single surgeon series, between 2005 and 2007, we operated on six consecutive patients with epileptic zones within the insula. The series included three men and three women, with a mean age of 50 months (range, 784 months). We excluded tumorous, hemorrhagic condition based on a previous study.

The six patients reported in this study were identified prospectively by a collaborative epilepsy team. All of the patients had drug-resistant seizures and each patient was evaluated with video scalp electroencephalography (EEG) monitoring to capture a typical seizure. In all cases, neuroimaging data included an optimal brain magnetic resonance imaging (MRI), [18F] fluorodeoxyglucose-positron emission tomography, and interictal single-photon emission computed tomography (SPECT). An ictal SPECT was performed for only three of the patients. We took postoperative brain MRIs within $48 \mathrm{~h}$ to confirm the extent of resection.

\section{Monitoring}

To determine which monitoring methods in insular epilepsy is most effective. We implanted strips after lobectomy in one patient, stereotactic depth electrodes in two patients, and open direct subpial depth electrodes in three patients.

An eight-contact, button-style strip (AD-TECH, Wisconsin) was used for chronic implantation in one patient. In two patients, a four-contact electrode $(2 \mathrm{~mm}$ in length, separated by $10 \mathrm{~mm}$ ) was inserted (right insular in one patient, left insular in the other) using a Leksell stereotactic frame via a posterior parietal route. The same depth electrodes were inserted subpially after frontotemporal craniotomy in the other three patients. Two of these patients were remonitored after a previous unsatisfactory seizure outcome, and the other patient was monitored just for insular cortex. Standard pterional craniotomies using the wide transsylvian approach with general anesthesia were used for all patients. Splitting the sylvian fissure along its full length is important for exposing the insular. The narrow surgical window, the lime recess, was exposed from the limen insular to the most lateral part of the lenticulostriate arteries under a microscope [21]. Great care was used to avoid injuring the lensticulostriate arteries and middle cerebral branches.
Depth electrodes were implanted subpially from the limen recess to the anterior insular point and posterior insular point using the Stryker navigation system (Stryker instruments, Kalamazoo, MI, USA). We performed imageguided implantation of the intracerebral depth electrodes and the electrodes could be left in place for up to 7 days. Each electrode had contacts of $2 \mathrm{~mm}$ length, separated by $10 \mathrm{~mm}$. Depth electrodes were implanted after wide sylvian dissection from the limen recess to the anterior insular point and posterior insular point using navigation guidance [22], with a minimum of two depth electrodes into the short insular gyrus and one into the long insular gyrus.

\section{Subpial depth electrodes insertion}

The patient was positioned supine with a $60^{\circ}$ head rotation and the sylvian fissure parallel to the floor. The head was held in place with a three-point skull fixation. The scalp incision was made in the frontotemporal area just like a dandy incision to cover the frontal and temporal area.

Opening the frontoorbital ramus using the opercular landmark provided access to the most anteroinferior portion of the insula. After wide opening of sylvian fissure under an operating microscope with standard technique, subarachnoid dissection was performed to trace from the middle cerebral artery branch until the most lateral part of the lenticulostriate arteries. A small pia incision was made in the limen recess just under the limen insulae. Repeated confirmation of the trajectory under navigation was required for optimal placement of the depth electrodes. The reported mean distance between the limit of the limen insulae and the junction of the anterior and superior limiting sulci was $31.80 \mathrm{~mm}$ [21]. A 4-mm contact depth and 10-mm spacing were enough to cover the short and long gyri (SD04RSP10X-000, AD-TECH, USA).

\section{Results}

Insular monitoring

For this study, we monitored six patients with insular lobe seizures (Table 1). Patient \#4 was monitored with chronic implantations using subdural grids due to uncontrolled seizures after frontal lobectomy. Patients \#5 and \#6 were monitored with one depth electrode implanted by a stereotactic procedure. But, only two contacts out of four were monitored after insertion of the depth electrodes (Fig. 1a). The other three patients (patients \#1, \#2, and \#3) were monitored using subpial depth electrodes. For each electrode, four contacts were inserted into the long insular gyrus and short insular gyrus (Fig. 1b,c). Twelve contacts under the insular gyrus were confirmed by postoperative 
Table 1 Presurgical evaluation, surgical treatment, and surgical outcome

\begin{tabular}{|c|c|c|c|c|c|c|c|c|c|c|c|}
\hline $\begin{array}{l}\text { Patient } \\
\text { no. }\end{array}$ & Age/sex & $\begin{array}{l}\text { Follow-up } \\
\text { (months) }\end{array}$ & $\begin{array}{l}\text { Seizure } \\
\text { focus }\end{array}$ & MRI & PET & EEG & Monitoring & $\begin{array}{l}\text { Insular } \\
\text { discharge }\end{array}$ & Operation & Pathology & $\begin{array}{l}\text { Seizure } \\
\text { outcome }^{\mathrm{a}}\end{array}$ \\
\hline 1 & 7 years/f & 6 & $\mathrm{R}$ & I & I & I & Subpial depth & + & $\mathrm{TI}$ & CD IIb & I \\
\hline 2 & 5 years $/ \mathrm{m}$ & 33 & $\mathrm{R}$ & $\mathrm{T}$ & $\mathrm{F}$ & $\mathrm{T}, \mathrm{I}$ & Subpial depth & + & $\mathrm{FL}, \mathrm{TL}+\mathrm{TI}$ & None & I \\
\hline 3 & 2 years $/ \mathrm{m}$ & 24 & $\mathrm{~L}$ & $\mathrm{~T}$ & $\mathrm{~F}, \mathrm{~T}$ & $\mathrm{~F}, \mathrm{~T}, \mathrm{I}$ & Subpial depth & + & $\mathrm{H}+\mathrm{TI}$ & NOS & I \\
\hline 4 & 7 months/f & 24 & $\mathrm{~L}$ & $\mathrm{~F}, \mathrm{I}$ & $\mathrm{F}, \mathrm{T}, \mathrm{I}$ & I & Strip & + & $\mathrm{FL}+\mathrm{PI}$ & $\mathrm{CD}$ & I \\
\hline 5 & $\begin{array}{l}4 \text { years } \\
5 \text { months/f }\end{array}$ & 11 & $\mathrm{R}$ & $\mathrm{F}$ & $\mathrm{F}$ & $\mathrm{F}, \mathrm{I}$ & Stereotactic depth & + & $\mathrm{FL}+\mathrm{PI}$ & $\begin{array}{l}\text { Hypertrophic } \\
\text { astrocyte }\end{array}$ & II \\
\hline 6 & 6 years $/ \mathrm{m}$ & 9 & $\mathrm{~L}$ & $\mathrm{~F}, \mathrm{I}$ & $\mathrm{T}$ & $\mathrm{F}, \mathrm{I}$ & Stereotactic depth & + & $\mathrm{TL}+\mathrm{PI}$ & MD & I \\
\hline
\end{tabular}

${ }^{\text {a }}$ Seizure outcome classified according to Engle's classification [7]

Sex: $m$ male, $f$ female; seizure focus-lateralization of the epileptogenic zone: $R$ right, $L$ left, $F$ frontal lobe, $F L$ frontal lobectomy, $H$ hemispherectomy, $I$ insular, $T$ temporal lobe, $T L$ temporal lobectomy, $T I$ total insulectomy, PI partial insulectomy; F18: interictal 18fluorodeoxyglucose positron emission tomography; SEEG electrodes - number and lateralization of intracranial stereotactically implanted electrodes: $R$ right, $L$ left; insular spikes - interictal spikes recorded in the insular cortex; $C D$ cortical dysplasia, $M D$ microdysgenesis

MRI. Two contacts were used in the depth electrodes to monitor the insular and five contacts were used for monitoring in the grid, but 12 contacts were used to monitor the long and short insular gyri.

\section{Surgical seizure outcome}

For the six patients (three men, three women), the mean age was 50 months (range, 7-84 months) and the mean followup period was 17.8 months (range 6-33 months). The six patients underwent partial or total insulectomy with or without previous epilepsy surgery. The site and extent of a previous cortisectomy is shown in Table 1. Of the three patients who had subpial depth electrodes, patient \#1 had the insular removed after monitoring, patient \#2 had an insulectomy about 8 months later due to uncontrolled seizure after frontal lobectomy (Fig. 2a,b), and patient \#3 underwent insulectomy due to uncontrolled seizure after hemispherectomy about 6 months later (Fig. 2c,d). The clinical outcome is summarized in Table 1.

Five patients were completely free of seizures (class I), with a mean follow-up of 17.8 months (range, 6 to 33 months). One patient was Engel class II after partial insulectomy with stereotactic depth electrode insertion.

A seizure-free outcome was achieved after complete insular removal in three out of the three patients $(100 \%)$, and after partial removal in two out of the three patients $(66.7 \%)$. Insular surgery was mostly performed under image guidance and tracing of depth electrodes using the suck-out technique (Fig. 3). Due to the small size of the specimen, we could only obtain insular pathology for only four out of six cases: two cases were cortical dysplasia, one was microdysgenesis, and one was hypertrophic astrocyte. It was difficult to obtain a pathological diagnosis


Fig. 1 Depth electrodes were inserted into the left insular, but only two contacts were used to monitor the insular cortex (a). Two depth electrodes were inserted subpially into the short insular gyrus and one into the long insular gyrus. Four contacts were used to monitor the insular cortex (b, c) 
for the remaining two cases due to the small size of the specimen.

\section{Discussion}

Insular monitoring

Results from our small series suggest that open direct subpial depth electrodes insertion may monitor insular more exactly and optimum guidance to insular surgery. Specifically, we could achieve good seizure outcome with this technique. We believe that this technique, which we monitored by an open direct subpial depth electrodes, is accurate because it is close to the insular cortex with multiple electrodes.

Despite the documented connections between the insular and the limbic structure, the role of the insular in the etiology of seizures is poorly understood [17]. Penfield has shown that there are similarities between the symptoms of a temporal lobe seizure and those evoked by insular cortex stimulation [16]. The role of the insular lobe in temporal lobe epilepsy remains unclear $[10,12,17]$, partly due to the fact that the insular cortex has long been considered inaccessible to depth EEG recording [12]. Because of its location deep within the sylvian fissure, scalp electroencephalography is relatively insensitive to the electrical activity within the insulae [17]. Chronic implantation of the insular with subdural electrodes requires stable placement of strip electrodes within a narrow space, which is hindered by middle cerebral branches and sylvian veins [3]. With stereotactic depth electrode insertion through the sylvian fissure, implantation must be carefully performed to protect the middle cerebral artery branches [3]. Stereotactic depth electrode placement into the insular cortex via a parietal route enables limited monitoring of a few insular gyrus, but does not fully cover the insular cortex.

Another chronic recording of the insular cortex with transopercular stereo-electroencephalography (SSEG), mostly with an electrode with a smaller diameter, allows for greater accuracy in the localization of the cortical targets using MRI and has been used to monitor the insular cortex $[12,20]$. SSEG is different from other electrode recordings, because SSEG allows the three-dimensional evaluation of the epileptogenic zone, which requires placement of the electrodes into different planes of the brain. SSEG has the advantage of a three-dimensional orientation and the ability to describe the mode of propagation for discharge spreading to or originating from the insula [12]. But, SSEG electrodes have to be removed after monitoring and therefore are not useful for guidance during insular removal.

Pia matter on the surface of the insular can be used to remove an insular tumor [13]. Tumor resection from under
Fig. 2 Strip insertion after frontal lobectomy due to recurrent epilepsy (a). Postoperative MRI shows complete removal of the insular (b). Insular monitoring and surgery were carried out after hemispherectomy due to recurrent epilepsy. The preoperative and postoperative MRI show that the remnant insular cortex was completely removed using subpial depth electrodes $(\mathbf{c}, \mathbf{d})$

the pia matter minimizes disturbances to the middle cerebral arteries [25]. Depth electrode insertion subpially after a small incision to the pia matter in the limen insular provides a surgical window for inserting depth electrodes, and the depth electrodes can be used as a surgical landmark for removal of the insular cortex without injury to adjacent vital structures.

The risks of insular surgery are not significant with respect to the development of the microsurgical anatomy and technique [21, 22]. Neurosurgical complications can be reduced and the extent of resection can be maximized for intrinsic insular tumors [13].

The distances between the inferior limit of the limen insulae and the anterior insular point or posterior insular point were previously reported $31.80 \mathrm{~mm}(27.36-35.61)$ and $34.40 \mathrm{~mm}$ (31.11-38.80), respectively [21]. Short insular gyrus and long insular gyrus can be fully covered in length with depth electrodes.

Neurovital structures, such as the internal capsule and basal ganglia, are located deep within the insular lobe and can be damaged during insular surgery. The average distance of the limen insulae to the putamen is only $5.7 \mathrm{~mm}$ [21]. The exact depth of the insular removal is difficult to control and monitor in such a small space. Blind cortisectomy deep into the basal ganglia could damage these vital structures. Therefore, cortisectomy using inserted subpial depth electrodes as a surgical landmark can prevent injury to the surrounding tissues. The insertion of depth electrodes and intraoperative navigation can provide appropriate orientation for insular surgery in a narrow space. The insertion of subpial depth electrodes in the insular allows the safe and efficient removal of the insular cortex without damaging the neurovital structure.

\section{Insular surgery}

In a review of previous reports, the additional removal of the insular after temporal lobectomy when the insular displayed interictal epileptiform activity on electrocography did not improve seizure outcome [18]. Further, seizures originating in the insular cortex are not influenced by temporal lobectomy, which likely explains the failure of surgical procedures in treating temporal lobe epilepsy [12].

Partial seizure can be completely resolved with resection of the insular lesion and surrounding cortex if the insulae are adequately monitored by standard chronic recording 

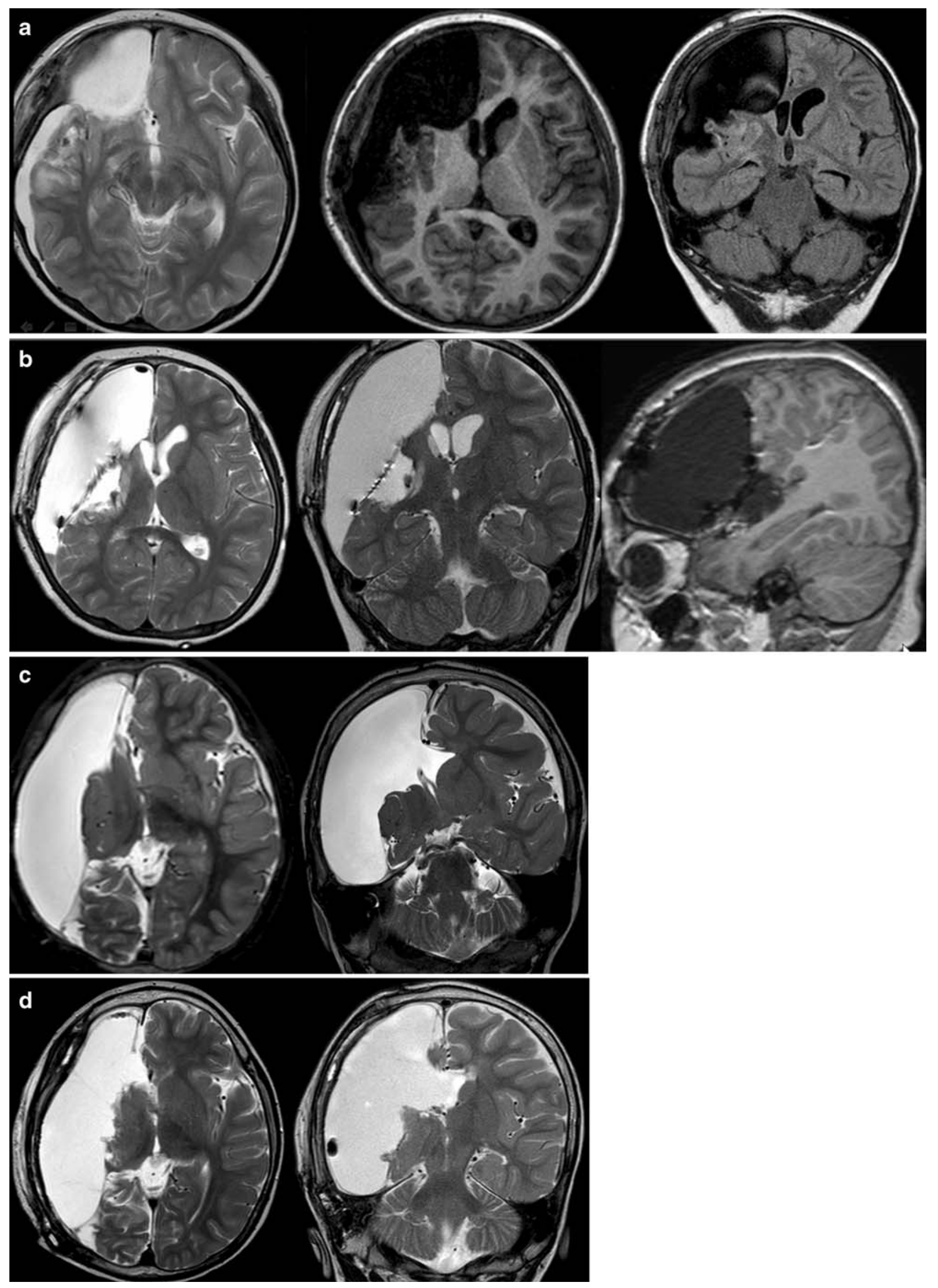

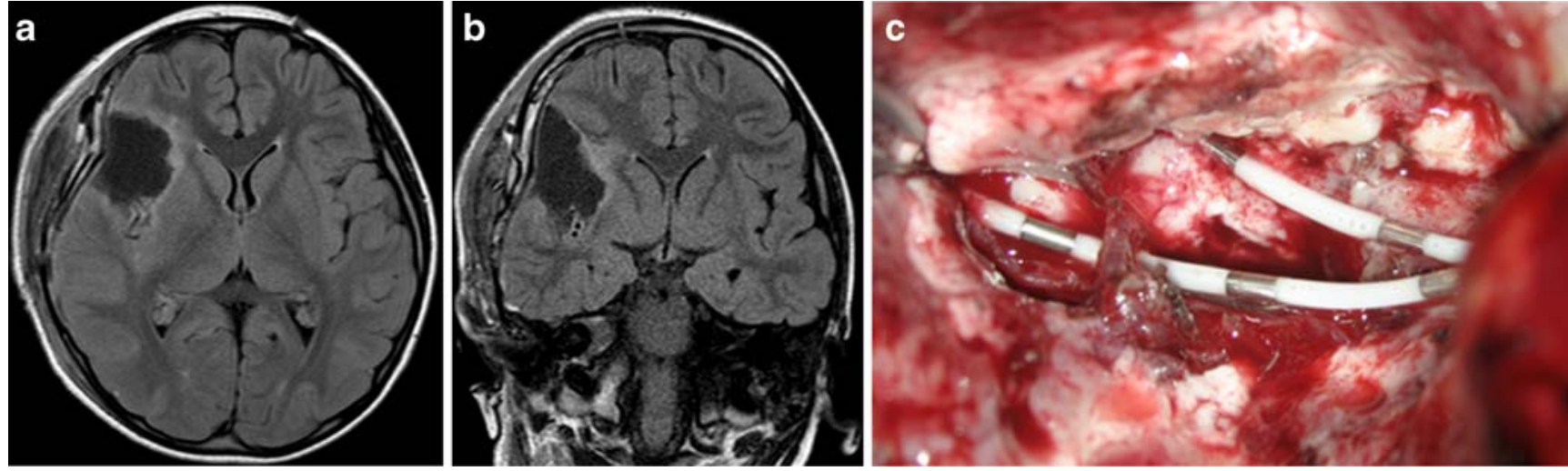

Fig. 3 Postoperative MRI shows the absence of the insular cortex without damage to the middle cerebral branches (a). b Cortisectomy can be carried out using the depth electrodes. During removal of the insular cortex, the contacts for depth and intraoperative navigation orient the surgeon within the insular cortex (c)

[17]. Also, non-lesional patients with insular seizure onset and nocturnal hypermotor seizure have successfully undergone epilepsy surgery [6].

Insular resection has been attempted to improve the surgical outcome after temporal lobectomy. Some insular seizures may be mistaken for temporolimbic or opercular seizures, which could in part explain the persistence of disabling seizures in $30 \%$ of patients after anterior temporal lobectomy [8]. Insulectomy is accompanied by a considerably higher incidence of neurological complication than temporal lobectomy alone. Therefore, insulectomy is not recommended even if there is an insular epileptiform abnormality on the postexcision cortical EEG [18].

Even after a complete functional hemispherectomy, postoperative seizures have been reported in up to $33 \%$ of patients $[19,23]$ such as incomplete disconnection or pathological condition to contralateral independent epileptogenic abnormalities [1, 4, 5, 9, 19].

The presence of insular tissue, which was intentionally preserved during surgery, did not correlate statistically with persistent seizures after hemispherectomy $(p=0.2)$ [9]. But, others have reported that the presence of the insular cortex is statistically associated with the presence of postoperative seizure $(p=0.021)$ after functional hemispherectomy [2]. Even with a small sample size, the role of the insular cortex should be considered [2].

Vertical parasagittal hemispherotomy, compared with the other three techniques of lateral hemispherotomy, allows a complete disconnection including the insular cortex when the lateral approaches require its removal [4].

Incomplete removal of the cortical structure in hemispherectomy could be hidden by the ipsilateral insular cortex. We performed the additional removal of the insular cortex after hemispherectomy in one case. We confirmed that the remnant insular was also epileptogenic using subpial insular grids. We recommend performing insular removal if complete disconnection was previously performed but not with contralateral independent epileptogenic abnormalities.

\section{Conclusions}

Based on our study of cases of insular epilepsy, imageguided subpial depth electrode insertion provides precise monitoring of seizures and serves as a surgical landmark for insular surgery, which prevents injury to the adjacent neurovital structure. When monitoring results are compatible with insular epilepsy, a total insulectomy, rather than partial insulectomy, should be performed to achieve the optimal seizure outcome.

Conflict of interest None.

Open Access This article is distributed under the terms of the Creative Commons Attribution Noncommercial License which permits any noncommercial use, distribution, and reproduction in any medium, provided the original author(s) and source are credited.

\section{References}

1. Carreno M, Wyllie E, Bingaman W, Kotagal P, Comair Y, Ruggieri P (2001) Seizure outcome after functional hemispherectomy for malformations of cortical development. Neurology 57:331-333

2. Cats EA, Kho KH, Van Nieuwenhuizen O, Van Veelen CW, Gosselaar PH, Van Rijen PC (2007) Seizure freedom after functional hemispherectomy and a possible role for the insular cortex: the Dutch experience. J Neurosurg 107:275-280 
3. Cukiert A, Forster C, Andrioli MS, Frayman L (1998) Insular epilepsy. Similarities to temporal lobe epilepsy. Case report. Arq Neuropsiquiatr 56:126-128

4. Delalande O, Bulteau C, Dellatolas G, Fohlen M, Jalin C, Buret V, Viguier D, Dorfmüller G, Jambaqué I (2007) Vertical parasagittal hemispherotomy: surgical procedures and clinical long-term outcomes in a population of 83 children. Neurosurg Online 60: ONS19-ONS32, discussion ONS32

5. Devlin AM, Cross JH, Harkness W, Chong WK, Harding B, Vargha-Khadem F, Neville BG (2003) Clinical outcomes of hemispherectomy for epilepsy in childhood and adolescence. Brain 126:556-566

6. Dobesberger J, Ortler M, Unterberger I, Walser G, Falkenstetter T, Bodner T, Benke T, Bale R, Fiegele T, Donnemiller E, Gotwald T, Trinka E (2008) Successful surgical treatment of insular epilepsy with nocturnal hypermotor seizures. Epilepsia 49:159-162

7. Engel J, Van Ness P, Rassmussen T, Ojemann L (1993) Outcome with respect to epileptic seizures. In: Engle J (ed) Surgical treatment of the epilepsies. Raven, New York, pp 609-621

8. Engel J Jr (1993) Update on surgical treatment of the epilepsies. Summary of the Second International Palm Desert Conference on the Surgical Treatment of the Epilepsies (1992). Neurology 43:1612-1617

9. Gonzalez-Martinez JA, Gupta A, Kotagal P, Lachhwani D, Wyllie E, Luders HO, Bingaman WE (2005) Hemispherectomy for catastrophic epilepsy in infants. Epilepsia 46:1518-1525

10. Hatashita S, Nitta T, Koga N, Sugimura J, Sakakibara T, Takagi S (1983) Traumatic aneurysm of the intracavernous carotid artery. A case report. Neurol Med Chir (Tokyo) 23:885-890

11. Heffez DS (1997) Stereotactic transsylvian, transinsular approach for deep-seated lesions. Surg Neurol 48:113-124

12. Isnard J, Guenot M, Ostrowsky K, Sindou M, Mauguiere F (2000) The role of the insular cortex in temporal lobe epilepsy. Ann Neurol 48:614-623

13. Lang FF, Olansen NE, DeMonte F, Gokaslan ZL, Holland EC, Kalhorn C, Sawaya R (2001) Surgical resection of intrinsic insular tumors: complication avoidance. J Neurosurg 95:638-650
14. Ostrowsky K, Isnard J, Ryvlin P, Guenot M, Fischer C, Mauguiere F (2000) Functional mapping of the insular cortex: clinical implication in temporal lobe epilepsy. Epilepsia 41:681-686

15. Penfield W, Faulk ME Jr (1955) The insula. Further observations on its function. Brain 78:445-470

16. Penfield WJW (1954) Epilepsy and the functional anatomy of the human brain. Little, Brown, Boston

17. Roper SN, Levesque MF, Sutherling WW, Engel J Jr (1993) Surgical treatment of partial epilepsy arising from the insular cortex. Report of two cases. J Neurosurg 79:266-269

18. Silfvenius H, Gloor P, Rasmussen T (1964) Evaluation of insular ablation in surgical treatment of temporal lobe epilepsy. Epilepsia $5: 307-320$

19. Smith SJ, Andermann F, Villemure JG, Rasmussen TB, Quesney LF (1991) Functional hemispherectomy: EEG findings, spiking from isolated brain postoperatively, and prediction of outcome. Neurology 41:1790-1794

20. Talairach JBJ (1973) Stereotaxic approach to epilepsy: methodology of anatomofunctional stereotaxic investigations. Prog Neurol Surg 5:297-354

21. Tanriover N, Rhoton AL Jr, Kawashima M, Ulm AJ, Yasuda A (2004) Microsurgical anatomy of the insula and the sylvian fissure. J Neurosurg 100:891-922

22. Ture U, Yasargil DC, Al-Mefty O, Yasargil MG (1999) Topographic anatomy of the insular region. J Neurosurg 90: 720-733

23. Vining EP, Freeman JM, Brandt J, Carson BS, Uematsu S (1993) Progressive unilateral encephalopathy of childhood (Rasmussen's syndrome): a reappraisal. Epilepsia 34:639-650

24. Wang L, Zhang MZ, Zhao JZ, Meng GL, Han XD (2004) Clinical features and minimally invasive surgery of insular lesions: report of 42 cases. Chin Med J (Engl) 117:1104-1108

25. Zentner J, Meyer B, Stangl A, Schramm J (1996) Intrinsic tumors of the insula: a prospective surgical study of 30 patients. J Neurosurg 85:263-271 